\title{
A study of optical solitons with Kerr and power law nonlinearities by He's variational principle
}

\section{Engin Topkara}

\section{Daniela Milovic}

\section{Amarendra K. Sarma}

\section{Fayequa Majid}

\section{Anjan Biswas \\ biswas.anjan@gmail.com}

Applied Mathematics Research Center, Department of Mathematical Sciences, Delaware State University, Dover, DE 19901-2277, USA

Faculty of Electronic Engineering, Department of Telecommunications, University of Nis, Aleksandra Medvedeva 14, 1800, Nis, Serbia

Department of Physics, Indian Institute of Technology, Guwahati, Guwahati-781039, India

Department of Mathematics, Alabama A \& M University, Normal, AL-35762, USA

Center for Research and Education in Optical Sciences and Applications, Applied Mathematics Research Center, Department of Mathematical Sciences, Delaware State University, Dover, DE 19901-2277, USA

This paper studies optical solitons, in presence of perturbation terms, by the aid of He's variational principle. The inter-modal dispersion, self-steepening and nonlinear dispersion are all treated as perturbation terms. Both Kerr law as well as power law nonlinearities are considered in this paper. [DOI: 10.2971/jeos.2009.09050]

Keywords: fiber measurements, optical communications, pulse propagation and temporal solitons, Kerr effect, nonlinear optics

\section{INTRODUCTION}

Optical solitons play a major role in large amount of data communication, through optical fibers, the veins of modern communication. Therefore, this is one of the major research topics in Nonlinear Optics. There has been a large amount of success in this area of research during the past few decades. Thus, today optical solitons are a reality in many areas across the globe including many continents, especially in Europe and Oceania.

The governing equation is the Nonlinear Schrödinger's equation (NLSE), that governs the propagation of solitons through optical fibers, through trans-continental and trans-oceanic distances [1]-[10]. There are many mathematical features to this equation that interests the Nonlinear Optics community. One such feature is the integrability aspect of this equation, especially in presence of perturbation terms. While there are many numerical solutions available to this equation, it is always nice and convenient to have an analytical solution to the perturbed NLSE. This paper will study the integrability of the NLSE in presence of perturbation terms.

The NLSE falls under the category of nonlinear evolution equations in the mathematical world. There are various techniques that have been developed in the past few decades to carry out the integration of these equations. Some of these techniques are Hirota's bilinear method, Lie symmetry method, $F$ expansion method, $G^{\prime} / G$ method, Riccati's equation method, soliton ansatz method and many others that even lead to multiple solutions [10]. In this paper, one such method will be used to carry out the integration of the perturbed NLSE. This is called He's variational principle (HVP) $[1,5,9]$.

\section{MATHEMATICAL ANALYSIS}

The dimensionless form of the NLSE in a non-Kerr law media is given by [4]-[10]

$$
i q_{t}+a q_{x x}+b F\left(|q|^{2}\right) q=0
$$

where $x$ and $t$ represents the spatial and temporal variables respectively. The first term is the evolution term, The second term is the group velocity dispersion and the third term is the nonlinear term where the function $F$ dictates the type of nonlinearity in question. The dependent variable $q$ represents the wave profile and is a complex valued function. Solitons are the result of a delicate balance between dispersion and nonlinearity.

In Eq. (1), $F$ is a real-valued algebraic nonlinear function and it is necessary to have the smoothness of the complex function $F\left(|q|^{2}\right) q: C \mapsto C$. Considering the complex plane $C$ as a twodimensional linear space $R^{2}$, the function $F\left(|q|^{2}\right) q$ is $k$ times continuously differentiable, so that $[4,5]$

$$
F\left(|q|^{2}\right) q \in \bigcup_{m, n=1}^{\infty} C^{k}\left((-n, n) \times(-m, m) ; R^{2}\right) .
$$




\subsection{Perturbation terms}

The perturbed NLSE that is going to be studied in this paper is given by [5]

$$
\begin{aligned}
i q_{t}+a q_{x x} & +b F\left(|q|^{2}\right) q \\
& =i \alpha q_{x}+i \lambda\left(|q|^{2 m} q\right)_{x}+i v\left(|q|^{2 m}\right)_{x} q
\end{aligned}
$$

where the perturbation terms are located on the right-hand side of the equation. Here, in Eq. (3), $\alpha$ is the coefficient of inter-modal dispersion, $\lambda$ is the coefficient of self-steepening term for short pulses (typically $\leq 100$ femto seconds), $v$ is the higher order dispersion coefficient [4]. The parameter $m$ on two of the terms in the right-hand side of Eq. (3) makes these terms fully nonlinear. The special case when $m=1$ was studied earlier in 2009 [4]. This perturbed NLSE is going to be studied via HVP, in this paper, for Kerr and power law nonlinearity, with fully nonlinear perturbation terms.

\section{HE'S VARIATIONAL PRINCIPLE}

In this section, HVP will be introduced. Subsequently, it will be applied to carry out the integration of Eq. (3) for Kerr and power laws of nonlinearity for $F$ in Eq. (1).

The starting point is the solitary wave ansatz that is given by

$$
q(x, t)=g(s) e^{i \phi},
$$

where $P=g(s)$ represents the shape of the pulse and

$$
\begin{aligned}
& s=x-v t, \\
& \phi=-\kappa x+\omega t+\theta .
\end{aligned}
$$

Here, $v$ is the velocity of the soliton, $\kappa$ is the frequency while $\omega$ is the soliton wave number and $\theta$ is the phase constant. Substituting this ansatz into Eq. (3) and decomposing into real and imaginary parts yields the following pair of relations, respectively

$$
-\left(\omega+a \kappa^{2}\right) g+a g^{\prime \prime}+b g F\left(g^{2}\right)=\alpha \kappa g+\lambda \kappa g^{2 m+1}
$$

and

$$
v+\alpha+2 a \kappa+\{(2 m+1) \lambda+2 m v\} g^{2 m}=0
$$

where the notations $g^{\prime}=d g / d s$ and $g^{\prime \prime}=d^{2} g / d s^{2}$ are used. Now, Eq. (8) yields

$$
g(s)=\left[-\frac{v+\alpha+2 a \kappa}{(2 m+1) \lambda+2 m v}\right]^{\frac{1}{2 m}}
$$

From Eq. (9), it is important to note that solitons will exist for

$$
(v+\alpha+2 a \kappa)\{(2 m+1) \lambda+2 m v\}<0
$$

This is the expression for the function $g(s)$ in terms of the soliton velocity $(v)$.

Now, multiplying both sides of the real part equation, given by Eq. (7), by $g^{\prime}$ and integrating yields

$$
\begin{aligned}
a\left(g^{\prime}\right)^{2}-\left(\omega+\alpha \kappa+a \kappa^{2}\right) g^{2} & +\frac{\lambda \kappa g^{2 m+2}}{m+1} \\
& +2 b \int g g^{\prime} F\left(g^{2}\right) d g=K
\end{aligned}
$$

where $K$ is a constant. The stationary integral $J$ is then defined as

$$
J=\int_{-\infty}^{\infty} K d s
$$

which, from Eq. (11), is therefore given by

$$
\begin{aligned}
J=\int_{-\infty}^{\infty}\left[a\left(\frac{d g}{d s}\right)^{2}\right. & -\left(\omega+\alpha \kappa+a \kappa^{2}\right) g^{2} \\
& \left.+\frac{\lambda \kappa g^{2 m+2}}{m+1}-2 b \int g g^{\prime} F\left(g^{2}\right) d g\right] d s
\end{aligned}
$$

Finally, the 1-soliton solution ansatz, given by

$$
g(s)=A f\left[\frac{1}{\cosh (B s)}\right]
$$

is substituted into Eq. (13). Here, in Eq. (14), the parameters $A$ and $B$ represent the amplitude and inverse width of the soliton respectively, and the functional $f$ depends on whether the nonlinear function $F$ is Kerr or power. He's semi-inverse variational principle states that the parameters $A$ and $B$ are determined from the solution of the equations $[1,5,9]$

$$
\frac{\partial J}{\partial A}=0
$$

and

$$
\frac{\partial J}{\partial B}=0
$$

The parameters $A$ and $B$ will now be determined for the following cases of nonlinearity in the following subsections.

\subsection{Kerr law}

The Kerr law of nonlinearity originates from the fact that a light wave in an optical fiber faces nonlinear responses from non-harmonic motion of electrons bound in molecules, caused by an external electric field. Even though the nonlinear responses are extremely weak, their effects appear in various ways over long distance of propagation that is measured in terms of light wavelength. The origin of nonlinear response is related to the non-harmonic motion of bound electrons under the influence of an applied field. As a result the induced polarization is not linear in the electric field, but involves higher order terms in electric field amplitude $[4,5]$.

In the case of Kerr law nonlinearity where $F(u)=u$, the perturbed NLSE is given by

$$
i q_{t}+a q_{x x}+b|q|^{2} q=i \alpha q_{x}+i \lambda\left(|q|^{2 m} q\right)_{x}+i v\left(|q|^{2 m}\right)_{x} q
$$

and therefore Eq. (7) reduces to

$$
a g^{\prime \prime}+b g^{3}-\left(\omega+a \kappa^{2}\right) g+\lambda \kappa g^{2 m+1}=0
$$

Thus, the stationary integral, from Eq. (17), is given by

$$
\begin{aligned}
J=\int_{-\infty}^{\infty}\left[a\left(\frac{d g}{d s}\right)^{2}-(\omega\right. & \left.+\alpha \kappa+a \kappa^{2}\right) g^{2} \\
& \left.+\frac{\lambda \kappa g^{2 m+2}}{m+1}-\frac{b}{2} g^{4}\right] d s
\end{aligned}
$$


For Kerr law nonlinearity, the appropriate form of the soliton is given by

$$
g(s)=\frac{A}{\cosh (B s)}
$$

and so J, from Eq. (19), simplifies to

$$
\begin{aligned}
J & =\frac{2 a}{3} A^{2} B+\frac{2 b A^{4}}{3 B}-2\left(\omega+\alpha \kappa+a \kappa^{2}\right) \frac{A^{2}}{B} \\
& =\frac{2 m \lambda \kappa}{(m+1)(2 m+1)} \frac{\Gamma(m) \Gamma\left(\frac{1}{2}\right)}{\Gamma\left(m+\frac{1}{2}\right)} \frac{A^{2 m+2}}{B}
\end{aligned}
$$

Equations (15) and (16) give the following pair of algebraic equations

$$
\begin{gathered}
\frac{a}{3} A B^{2}+\frac{2 b A^{3}}{3}-\left(\omega+\alpha \kappa+a \kappa^{2}\right) A \\
+\frac{m \lambda \kappa}{2 m+1} \frac{\Gamma(m) \Gamma\left(\frac{1}{2}\right)}{\Gamma\left(m+\frac{1}{2}\right)} A^{2 m+1}=0 \\
\frac{a}{3} A B^{2}-\frac{b A^{3}}{3}-\left(\omega+\alpha \kappa+a \kappa^{2}\right) A \\
-\frac{m \lambda \kappa}{(m+1)(2 m+1)} \frac{\Gamma(m) \Gamma\left(\frac{1}{2}\right)}{\Gamma\left(m+\frac{1}{2}\right)} A^{2 m+1}=0
\end{gathered}
$$

From Eqs. (22) and (23), the equation for the soliton amplitude $A$ is given by

$$
\begin{aligned}
b A^{2}+\frac{m(m+2) \lambda \kappa}{(m+1)(2 m+1)} & \frac{\Gamma(m) \Gamma\left(\frac{1}{2}\right)}{\Gamma\left(m+\frac{1}{2}\right)} A^{2 m} \\
& =2\left(\omega+\alpha \kappa+a \kappa^{2}\right)
\end{aligned}
$$

while the inverse width $B$ is obtained from

$$
\begin{aligned}
B=\left[\frac{b A^{2}}{a}-\frac{3}{a}\right. & \left(\omega+\alpha \kappa+a \kappa^{2}\right) \\
& \left.\quad+\frac{3 m \lambda \kappa}{a(m+1)(2 m+1)} \frac{\Gamma(m) \Gamma\left(\frac{1}{2}\right)}{\Gamma\left(m+\frac{1}{2}\right)} A^{2 m}\right]^{\frac{1}{2}}
\end{aligned}
$$

The special case for $m=1$ with $\alpha=0$ was solved in 2009 [5]. From Eq. (25), it is possible to observe that it is necessary to have

$$
a>0
$$

and

$$
m>0
$$

for the solitons to exist.

\subsection{Power law}

This law of nonlinearity arises in nonlinear plasmas that solves the problem of small $\mathrm{K}$-condensation in weak turbulence theory. It also arises in the context of nonlinear optics. Physically, various materials, including semiconductors, exhibit power law nonlinearities [4, 5].
For the case of power law nonlinearity, where $F(u)=u^{n}$, the perturbed NLSE is given by

$i q_{t}+a q_{x x}+b|q|^{2 n} q=i \alpha q_{x}+i \lambda\left(|q|^{2 m} q\right)_{x}+i v\left(|q|^{2 m}\right)_{x} q$

In Eq. (28), the parameter $n$ dictates the power law parameter. The special case with $n=1$ reduces to Kerr law nonlinearity. For power law nonlinearity, it is necessary to have $0<n<2$ to prevent wave collapse $[1,4,5]$ and, in particular, $n \neq 2$ to avoid self-focusing singularity [4]. Thus, Eq. (7) reduces to

$$
a g^{\prime \prime}+b g^{2 n+1}-\left(\omega+a \kappa^{2}\right) g+\lambda \kappa g^{2 m+1}=0
$$

In this case, therefore, the stationary integral Eq. (13) is given by

$$
\begin{aligned}
J=\int_{-\infty}^{\infty}\left[a\left(\frac{d g}{d s}\right)^{2}-(\right. & \left.\omega+\alpha \kappa+a \kappa^{2}\right) g^{2} \\
& \left.-\frac{b g^{2 n+2}}{n+1}+\frac{\lambda \kappa g^{2 m+2}}{m+1}\right] d s
\end{aligned}
$$

For power law nonlinearity, the hypothesis

$$
g(s)=\frac{A}{\cosh ^{\frac{1}{n}}(B s)}
$$

simplifies $J$ to

$$
\begin{gathered}
J=\left\{\frac{a A^{2} B}{n(n+2)}-\left(\omega+\alpha \kappa+a \kappa^{2}\right) \frac{A^{2}}{B}\right. \\
\left.+\frac{2 b A^{2 n+2}}{(n+1)(n+2) B}\right\} \frac{\Gamma\left(\frac{1}{n}\right) \Gamma\left(\frac{1}{2}\right)}{\Gamma\left(\frac{1}{n}+\frac{1}{2}\right)} \\
+\frac{\lambda \kappa}{m+1} \frac{\Gamma\left(\frac{m+1}{n}\right) \Gamma\left(\frac{1}{2}\right)}{\Gamma\left(\frac{m+1}{n}+\frac{1}{2}\right)} \frac{A^{2 m+2}}{B}
\end{gathered}
$$

By HVP, the two equations from Eqs. (15) and (16) are

$$
\begin{aligned}
& \left\{\frac{a B^{2}}{n(n+2)}-\left(\omega+\alpha \kappa+a \kappa^{2}\right)+\frac{2 b A^{2 n}}{n+2}\right\} \\
& +\lambda \kappa A^{2 m} \frac{\Gamma\left(\frac{1}{n}+\frac{1}{2}\right)}{\Gamma\left(\frac{1}{n}\right)} \frac{\Gamma\left(\frac{1}{n}+\frac{m}{n}\right)}{\Gamma\left(\frac{1}{n}+\frac{1}{2}+\frac{m}{n}\right)}=0
\end{aligned}
$$

and

$$
\begin{array}{r}
\left\{\frac{a B^{2}}{n(n+2)}-\left(\omega+\alpha \kappa+a \kappa^{2}\right)+\frac{2 b A^{2 n}}{(n+1)(n+2)}\right\} \\
+\frac{\lambda \kappa A^{2 m}}{m+1} \frac{\Gamma\left(\frac{1}{n}+\frac{1}{2}\right)}{\Gamma\left(\frac{1}{n}\right)} \frac{\Gamma\left(\frac{1}{n}+\frac{m}{n}\right)}{\Gamma\left(\frac{1}{n}+\frac{1}{2}+\frac{m}{n}\right)}=0
\end{array}
$$

respectively. From Eqs (33) and (34) eliminating $B$ gives

$$
\begin{array}{r}
\frac{(m+2) \lambda \kappa A^{2 m}}{2(m+1)} \frac{\Gamma\left(\frac{1}{n}+\frac{1}{2}\right)}{\Gamma\left(\frac{1}{n}\right)} \frac{\Gamma\left(\frac{1}{n}+\frac{m}{n}\right)}{\Gamma\left(\frac{1}{n}+\frac{1}{2}+\frac{m}{n}\right)}+\frac{b A^{2 n}}{n+1} \\
=\omega+\alpha \kappa+a \kappa^{2}
\end{array}
$$

which is the algebraic equation for obtaining amplitude $A$ with power law nonlinearity. After obtaining the amplitude 
$A$ from Eq. (35), the inverse width $B$ can be obtained from

$$
\begin{aligned}
B= & {\left[\frac{n(n+2)}{a}\left(\omega+\alpha \kappa+a \kappa^{2}\right)-\frac{2 n b A^{2 n}}{a}\right.} \\
& \left.-\frac{n(n+2) \lambda \kappa A^{2 m}}{a} \frac{\Gamma\left(\frac{1}{n}+\frac{1}{2}\right)}{\Gamma\left(\frac{1}{n}\right)} \frac{\Gamma\left(\frac{1}{n}+\frac{m}{n}\right)}{\Gamma\left(\frac{1}{n}+\frac{1}{2}+\frac{m}{n}\right)}\right]^{\frac{1}{2}}
\end{aligned}
$$

which is obtained by eliminating the amplitude $A$ between Eqs. (33) and (34). From Eq. (36), it is again possible to conclude that solitons will exist provided the same condition, as seen Eq. (26), is valid.

\section{CONCLUSIONS}

In this paper, the He's semi-inverse variational principle is used to carry out the integration of the NLSE with a few Hamiltonian perturbation terms and the Raman scattering term. Both the Kerr law as well as the power law nonlinearities are considered. After obtaining the 1-soliton solutions with these two kind of nonlinearities, the parameter domains are also identified for the soliton solution to exist. These results are from purely analytical studies and thus a closed form soliton solution has been obtained.

\section{ACKNOWLEDGMENTS}

The research work of the first author is fully funded by Army Grant Number DAAD 19-03-1-0375 and this support is very thankfully acknowledged.

The research work of the fifth author $(A B)$ was fully supported by NSF-CREST Grant No: HRD-0630388 and this support is genuinely and sincerely appreciated.

\section{References}

[1] A. Biswas, "Temporal 1-soliton solution of the complex GinzburgLandau equation with power law nonlinearity" Prog. Electromagn. Res. 96, 1-7 (2009).

[2] J. H. He, "Variational iteration method- a kind of non-linear analytical technique: some examples" Int. J. Nonlinear Mech. 34, 699-708 (1999).

[3] J. H. He, "Variational principles for some nonlinear partial differential equations with variable coefficients" Chaos Soliton. Fract. 99, 847-851 (2004).

[4] R. Kohl, D. Milovic, E. Zerrad, and A. Biswas, "Optical soliton perturbation in a non-Kerr law media" Opt. Laser Technol. 40, 647-662 (2008).

[5] R. Kohl, A. Biswas, D. Milovic, and E. Zerrad, "Optical solitons by He's variational principle in a non-Kerr law media" J. Infrared Millim. Te. 30, 526-537 (2009).

[6] M. Mishra, and S. Konar, "High bit rate dense dispersion-managed optical communication systems with distributed amplification" Prog. Electromagn. Res. 78, 301-320 (2008).

[7] A. K. Sarma, "Dark soliton switching in an NLDC in the presence of higher-order perturbative effects" Opt. Laser Technol. 41, 247-250 (2009).

[8] S. Shwetanshumala, S. Jana, and S. Konar, "Propagation of a mixture of modes of a laser beam in a medium with saturable nonlinearity" J. Electromagnet. Wave. 20, 65-77 (2006).

[9] S. Shwetanshumala, "Temporal solitons of modified complex Ginzburg-Landau Equation" Prog. Electromagn. Res. Lett. 3, 17-24 (2008).

[10] A. M. Wazwaz, "Multiple soliton solutions for a (2+1)-dimensional integrable KdV6 equation" to appear in Commun. Nonlinear. Sci. 